\title{
INDICADOR DE ARBORIZAÇÃO URBANA COMO APOIO AO PLANEJAMENTO DE CIDADES BRASILEIRAS
}

\author{
Allan Yu Iwama ${ }^{1}$
}

\section{RESUMO}

O estudo teve o objetivo de aplicar o método de amostragem simples para a elaboração de um índice de arborização baseado no número de árvores por quilômetro de calçada (arv/km). Os dados foram levantados em duas cidades do oeste paulista, Martinópolis e Pirapozinho, para estimar o número total de árvores e de quilômetro linear percorrido. Baseado nesses resultados e em pesquisas sobre os trabalhos de arborização urbana foi feita uma análise dos municípios baseada em um indicador espacial de arv/km, indicando aqueles em situação mais crítica (<20 arv/km) e menos crítica (> $80 \mathrm{arv} / \mathrm{km}$ ) em relação ao total analisado $(\mathrm{n}=28)$. Os resultados podem auxiliar na construção de um indicador espacial para o planejamento regional da arborização urbana nas cidades, considerando pelos menos quatro elementos: universo amostral, a unidade amostral (tamanho e forma), aspectos qualitativos de arborização (estrutura e composição das espécies arbóreas, entre outros) e o padrão de distribuição de bairros (e padrões de construção) e determinados usos urbanísticos.

Palavras-chave: Amostragem; Indicador espacial; Meio urbano.

\section{URBAN FOREST INDEX AS SUPPORT TO BRAZILIAN URBAN PLANNING}

\section{ABSTRACT}

The study applied a sampling method for the construction of an urban forest index based on the number of trees per linear kilometers (arv/km). The collected data were realized in two cities of western São Paulo, Martinópolis and Pirapozinho to estimate the total number of trees for linear kilometer. Based on these results and research on the urban forest an

Recebido em 05.12.2013 e aceito em 03.04.2014

${ }^{1}$ Engenheiro Ambiental, mestre em Sensoriamento Remoto e doutorando em Ambiente e Sociedade pelo Núcleo de Estudos e Pesquisas Ambientais da Unicamp (NEPAM/UNICAMP). E-mail: allan.iwama@gmail.com 
analysis was made of the municipalities based on the spatial indicator of arv $/ \mathrm{km}$, indicating those in most critical situation ( $<20 \mathrm{arv} / \mathrm{km})$ and less critical ( $>80 \mathrm{arv} / \mathrm{km})$ in relation to the total analyzed $(n=28)$. The results can support in a construction of a spatial index for regional planning of urban forest in cities, considering at least four elements: the sample universe, the sampling unit (size and shape), qualitative aspects of urban forest (structure and species composition trees) and the patterns of distribution of districts (and building areas) and urban uses.

Keywords: Statistical Sampling; Spatial indicator; Urban.

\section{INTRODUÇÃO}

Os espaços verdes e a arborização urbana podem desempenhar um papel fundamental na melhoria da qualidade de vida nas áreas urbanas (GREY; DENEKE, 1978; CAVALHEIRO; DEL PICCHIA, 1992; GRAZIANO, 1994; MILANO; DALCIN, 2000). Em particular, a arborização urbana pode ser considerada um importante recurso para a manutenção do espaço urbano, desempenhando uma importante função para a diversidade da fauna e de diferentes paisagens urbanas, contribuindo dessa forma para a qualidade ambiental do ambiente urbano (BEATLEY; MANNING, 1997; BOLUND; HUNHAMMAR, 1999; MASCARÓ, 1996; SOARES, 1998).

Diversos estudos têm indicado os benefícios ambientais e sociais da arborização urbana (GRAY; DENEKE, 1978; LAURIE, 1979; MILLER, 1988). Entre outros benefícios, podem ser destacados aqueles associados com a regulação do microclima, filtragem do ar e redução de nível ruído, controle de erosão e a redução dos níveis de estresse humano (ULRICH, 1984; SANDERS, 1986; SHEETS; MANZER, 1991; CAVALHEIRO; DEL PICCHIA, 1992; MILANO; DALCIN, 2000; PEDLOWSKI et al. 2002; BOLUND; HUNHAMMAR, 1999; McPHERSON et al., 2005; NOWAK; DWYER, 2007; ESCOBEDO; CHACALO, 2008).

Além de considerar os benefícios da arborização, diversos esforços têm buscado quantificar os serviços ambientais da arborização urbana (SILVA FILHO et al., 2002). Esses esforços vêm se direcionando, sobretudo, para os aspectos mais difíceis de obter um valor no mercado, como aqueles associados com aspectos psicológicos, nos quais envolvem a satisfação das pessoas em contato com o "verde" no ambiente urbano (SANTINI, 1993; GRAZIANO, 1994), muitas vezes vinculadas aos valores de mercado e de propriedade 
(PAYNE; STROM, 1975; DOMBROW et al., 2000) e de difícil mensuração. Apesar de sua importância, a arborização nas cidades tem sido frequentemente realizada sem um adequado planejamento no Brasil, muitas vezes ocasionando riscos de acidentes causados pelas quedas de galhos e copas das árvores na rede elétrica, trazendo custos crescentes com a manutenção de fios e cabos; ou raízes que causam danos ao pavimento da calçada, comprometendo a acessibilidade de portadores de mobilidade reduzida.

Dada a importância da arborização urbana, o presente estudo adotou um método de amostragem aleatória simples, considerando a quadra como unidade amostral, baseado em Rachid e Couto (1999).

\section{MATERIAIS E MÉTODOS}

\section{Estimativa de árvores por quilômetro: Martinópolis e Pirapozinho (SP)}

A metodologia adotada foi o levantamento de elementos arbóreos nas áreas urbanas das duas cidades, Martinópolis e Pirapozinho (Figura 1 que mostra a localização da área de estudo), utilizando o método de amostragem simples. Para isso foi realizado um sorteio aleatório das quadras em cada área sede, seguido de um levantamento quantitativo das árvores do entorno.

Figura 1. Mapa da área de estudo: área urbana de Martinópolis e de Pirapozinho, oeste do estado de São Paulo

Figure 1. Study area maps: Martinópolis and Pirapozinho urban areas, west of São Paulo

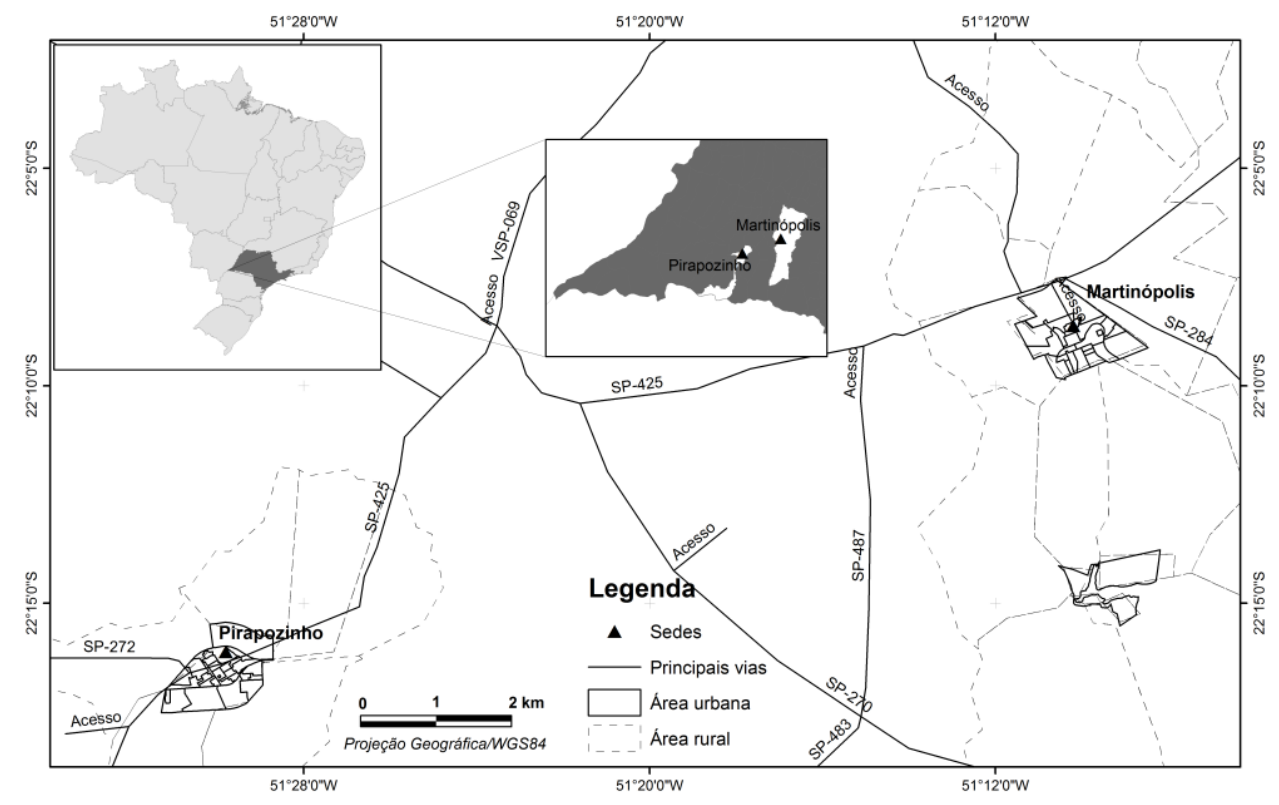


Os materiais utilizados para o levantamento foram: a carta oficial de Martinópolis, de 2005, na escala 1:7.500; carta oficial de Pirapozinho, de 2000, na escala 1:12.000; fotografia aérea da área sede de Pirapozinho de 2004; trena. A quadra foi considerada a unidade amostral. Após determinar o total de quadras de cada cidade, procedeu-se ao sorteio de uma amostra piloto, para registrar o número de elementos arbóreos, o total de quilômetros de calçada e a área ocupada pelas árvores consideradas no levantamento. Para estimar o número total de árvores por quilômetro foram feitas medidas do comprimento das quadras, largura da calçada de cada quadra e a metade da largura da rua (ver método proposto por Rachid e Couto, 1999), a partir de dados georreferenciados de cartas oficiais de arruamento e levantamento de campo. A Figura 2 apresenta as quadras com diferentes padrões e geometrias encontradas em campo (ver Figuras 2(a) e 2(b) - Pirapozinho e 2(c) Martinópolis) e o procedimento efetuado para o cálculo das variáveis mencionadas anteriormente (ver Figuras 2(d) - e 2(e) - a área total pertencente ao quarteirão).

Figura 2. (a) Quadras regulares e (b) irregulares da cidade de Pirapozinho/SP e de (c) Martinópolis, (d) Representação da quadra com medições efetuadas em campo: I - Comprimento da quadra; II - Largura média da calçada; III - Largura da metade da rua; (e) Representação da área total de um quarteirão (área hachurada)

Figure 2. (a) Regular and (b) irregular blocks in Pirapozinho cityand (c) Martinópolis city; (d) Blockrepresentationwith in loco measuring: I - Lengthofblock; II Averagewidthofsidewalk; III - Halfthewidthofthestreet; (e) Representationof a block total area (shadowarea)

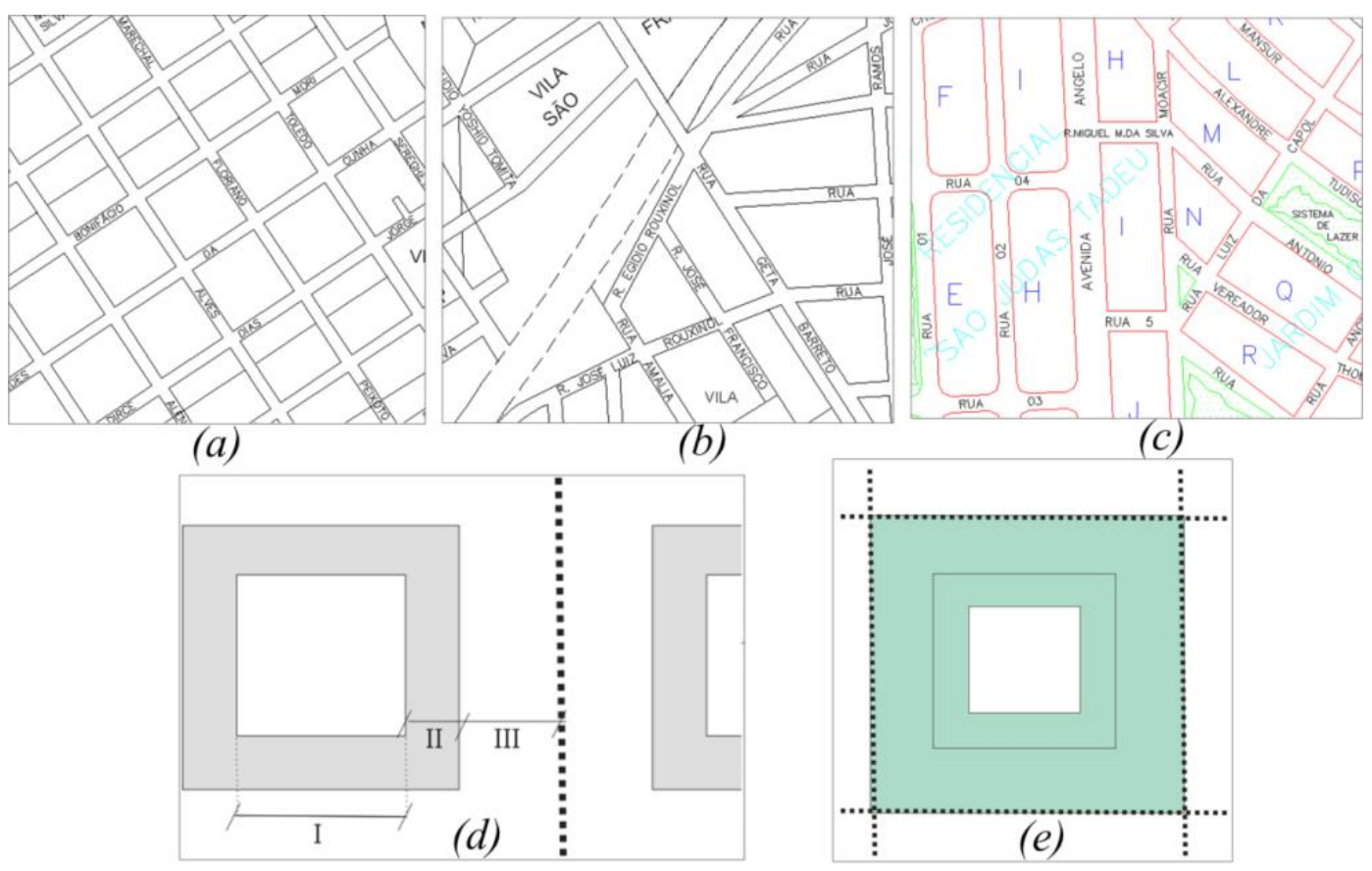


No total de árvores foram considerados todos os elementos arbóreos, arbustivos e herbáceos encontrados exclusivamente nas calçadas, ou seja, em área de domínio público. Após determinar todos os dados representativos para o estudo, foram calculadas a média $(\mathrm{x})$, variância $\left(\mathrm{S}^{2}\right)$ e o desvio padrão $(\mathrm{S})$. Considerou-se o conjunto de dados uma distribuição normal com intervalo de confiança (IC) de $95 \%$, tendo $N$ como o total de quarteirões, $n$ como o número de quarteirões selecionados na amostra (amostra piloto). Para estimar a razão populacional foram utilizados os estimadores definidos por Cochran (1977) - ver equação 1:

$$
R=\frac{X_{T}}{Y_{T}}(\text { Equação 1; Equation 1) }
$$

Onde $X_{T}$ é o valor "número de árvores em cada quarteirão" e $Y_{T}$ o valor "total de quilômetros (e de hectares) de calçada pertencente a cada quarteirão". O tamanho da amostra foi definido de forma a garantir que o número de unidades amostrais selecionadas tivesse um erro estatístico igual ou menor a 10\% em um intervalo de confiança de 95\%, obtendo-se amostra em torno de 9,7\% a 12,7\%. Alguns trabalhos têm definido o tamanho de amostra em torno desse percentual: amostra em 10\% (RACHID; COUTO; 1999; ROSSATTO et al., 2008); em 14,9\% (MENEGHETTI, 2003).

O erro relativo foi obtido pela equação 2: $d^{2}=\frac{z_{x \times f_{2} c v^{2}}^{x}(N-n)}{n \cdot N}$ (Equação 2; Equation 2)

Sendo $d^{2}$ : erro relativo; $z_{\alpha / 2}^{2}$ : valor encontrado na tabela de distribuição normal, com IC de 95\%; $c v^{2}$ : coeficiente de variação.

\section{Comparação de resultados: indicador de árvores por quilômetro}

A fim de obter um parâmetro de comparação, os resultados obtidos para as duas cidades do interior paulista foram confrontados com outros trabalhos realizados no período entre 1990 e 2010. No total foram analisados 28 trabalhos que continham o uso de indicadores de árvores por quilômetro de calçada e relação ao tema de análise qualiquantitativa de arborização urbana. Com base nessa análise e auxílio de um Sistema de Informação Geográfica (SIG), foram selecionados os municípios e distribuídos espacialmente de acordo com o indicador "árvores por quilômetro". A consulta de trabalhos sobre o tema não foi esgotada, mas teve o objetivo de trazer algumas reflexões sobre o uso de indicadores espaciais para auxiliar em uma análise de planejamento no âmbito regional. A seguir são apresentados os principais trabalhos analisados (Tabela 1). 
Tabela 1. Trabalhos analisados com o uso do indicador "árvore por quilômetro - arv/km"

Table 1. Studiesanalyzedbasedonindicatorsby 'treebykilometers - tree/km'

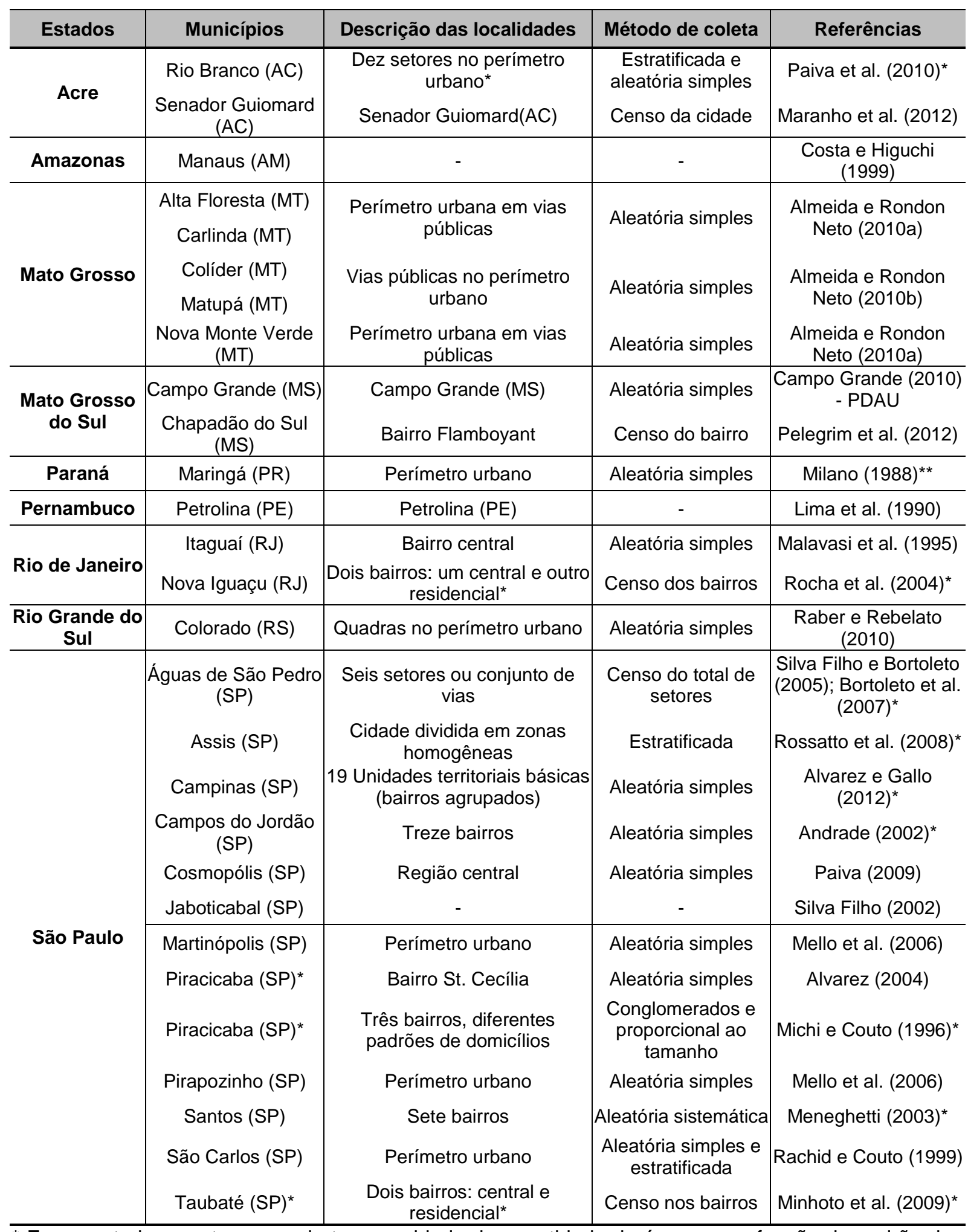

* Esses estudos mostram uma heterogeneidade da quantidade de árvores em função do padrão dos domicílios e bairros e do tipo de uso: comercial, residencial ou industrial. ${ }^{* *}$ Método baseado na amostragem de grades regulares de $200 \times 500 \mathrm{~m}$. Estimativa atualizada com 93.261 árvores (SAMPAIO e DE ANGELIS, 2008) 


\section{RESULTADOS E DISCUSSÃO}

Os resultados obtidos para as cidades de Martinópolis e Pirapozinho foram, respectivamente, um total de 6.397 e 4.301 árvores distribuídas no perímetro urbano, obtendo-se resultados de 53,45 e 34,10 árvores por quilômetro de calçada (arv/km). Esses resultados (MELLO et al., 2006) serviram de subsídios aos planos diretores municipais no âmbito de um convênio entre as prefeituras e FCT-UNESP, momento em que foi formado um grupo multidisciplinar para os estudos ambientais e urbanísticos das cidades em atendimento à exigência do Estatuto da Cidade (BRASIL, 2001 - Lei Federal 10.257/2001). Baseado nesses resultados e em pesquisas sobre os trabalhos de arborização urbana com uso do indicador árvores por quilômetro foi feita uma análise que permitiu indicar e localizar espacialmente os municípios em situação mais e menos crítica em relação ao total analisado $(\mathrm{n}=28)$. Analisando o indicador (arv $/ \mathrm{km})$ no conjunto de trabalhos selecionados, observou-se que os municípios situados no estado do Acre são aqueles com valores relativos mais críticos em relação aos outros municípios (menor do que 10 árvores por quilometro de calçada) - ver Figura 3(a). No outro extremo, pode-se observar os municípios de Águas de São Pedro-SP, Jaboticabal-SP, Itaguaí-RJ e Chapadão do Sul-MS (situados no Centro-Sul federativo), com valores superiores a 100 árvores por quilômetro (Figura 3(b)).

Figura 3. Municípios analisados situados (a) na região ao norte, (b) situados na região centro-sul do Brasil, (c) Frequência de cidades (e estados) por faixas de árv./ km

Figure 3. Municipalities in (a) North region, (b) Central-South regionofBrazil, (c) Frequencyofcitiesandstatesbyintervalsoftrees for kilometers
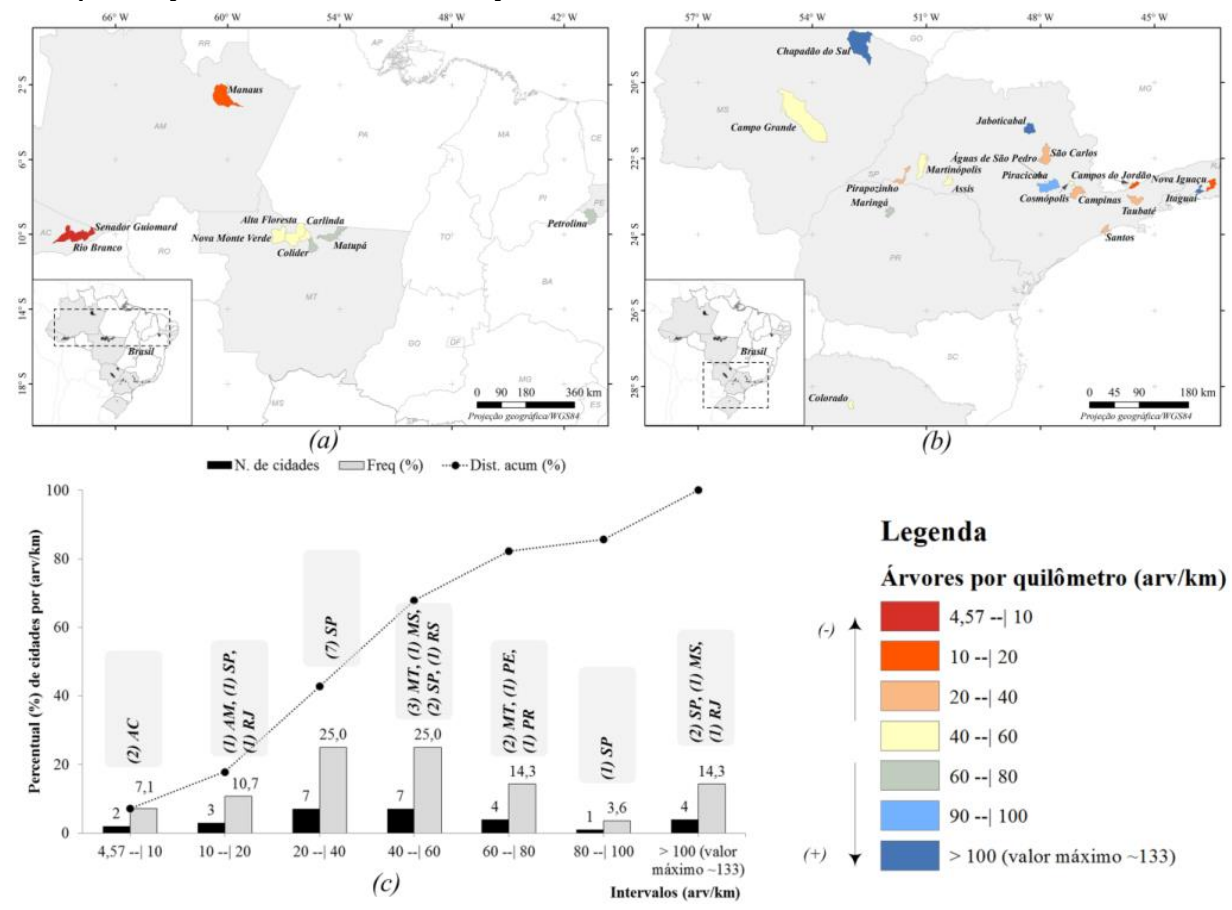
Analisando os intervalos de valores do indicador (arv/ $\mathrm{km}$ ), do total de 28 municípios, cerca de $25 \%$ estão nas faixas entre 20 -| 40 (inclui Pirapozinho) ou $40-\mid 60$ (inclui Martinópolis), ou seja, em valores intermediários. Na Figura 3(a) e 3(b) pode-se observar os municípios dentro dessas faixas de valores e sua localização espacial e na Figura 3(c) observa-se a frequência dos municípios por estados em cada faixa.

Tendo em vista a importância de se estabelecer um parâmetro de comparação do indicador árvores por quilômetro, esse trabalho sugere uma análise que seja orientada pelo grau de atenção ou alerta em relação a manutenção de árvores nos espaços públicos quanto aos aspectos de arborização urbana e seus benefícios: (1)> 100 árvores $/ \mathrm{km} \rightarrow$ baixa atenção - ainda que trabalhos sugiram que 100 árvores a cada quilômetro ou 10 a cada 1 metro seja um valor indicado para uma adequada arborização urbana (MILANO; DALCIN, 2000; PAIVA, 2009; PAIVA et al., 2010), essa situação não significa deixar de observar os critérios já mencionados nesse trabalho. Pelo contrário, é necessário observar a manutenção desses valores em termos quantitativos e qualitativos; (2) 60 a 100 árvores/km $\rightarrow$ média atenção; (3) 40 a 60 árvores $/ \mathrm{km} \rightarrow$ alta atenção; (4)10 a 40 árvores $/ \mathrm{km} \rightarrow$ muito alta atenção, (5)< 10 árvores/km $\rightarrow$ extremamente alta atenção.

Considerando esse grau de atenção de acordo com número de árvores/km, observase que as cidades do oeste paulista analisadas por Mello et al. (2006), Martinópolis e Pirapozinho, estão nas faixas de alta atenção e muita alta atenção quanto aos aspectos da quantidade e qualidade da arborização urbana (ver Figura 4 o ranqueamento dos municípios analisados).

Figura 4. Municípios analisados (total de 28) ranqueados em ordem decrescente do número de árvores $/ \mathrm{km}$. * Resultados de pesquisas predominantemente em bairros

Figure 4. Municipalitiesanalyzed (28 total) ranking bydecreasingofnumberoftree/km. * Researchresults in districts

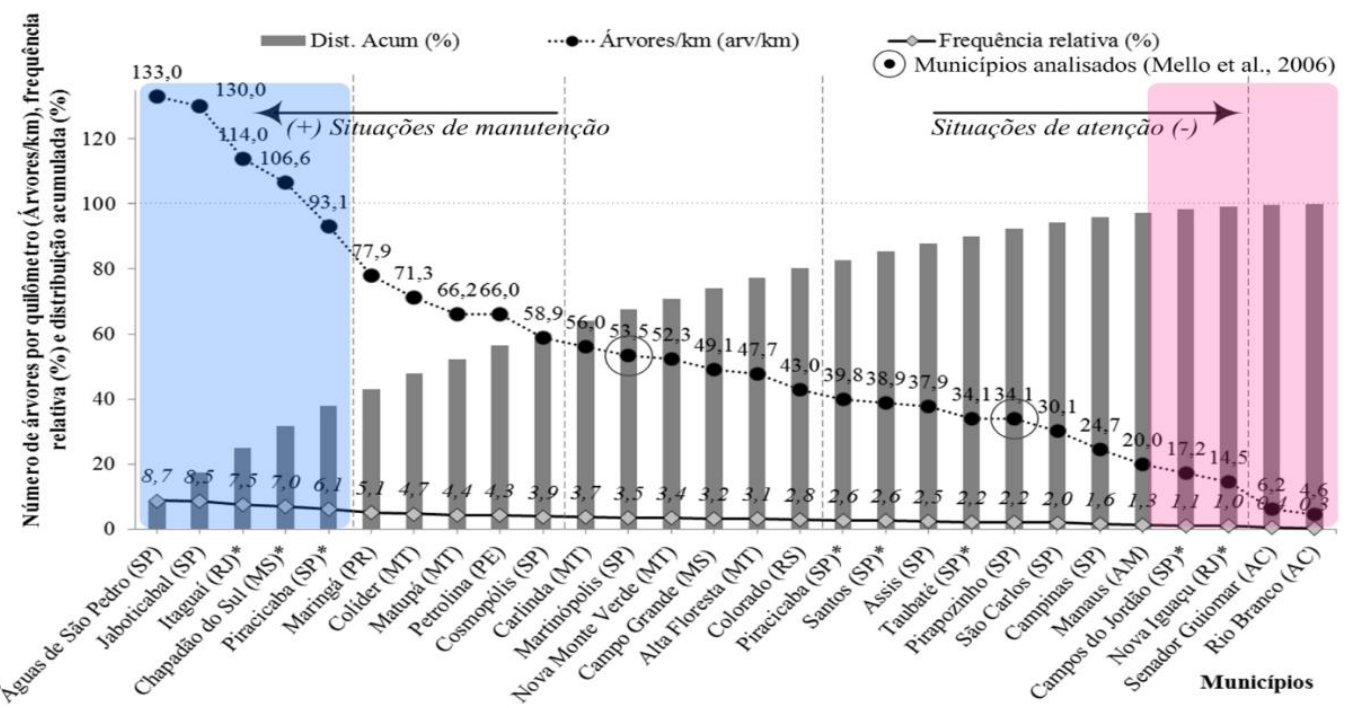


Essa análise, se por um lado permitiu identificar os municípios que necessitam de maior atenção em seus planos urbanísticos e de arborização urbana em relação à distribuição de árvores em calcadas públicas e/ou canteiros centrais, por outro necessita que alguns elementos sejam destacados. O primeiro elemento se trata do universo amostral. Em uma análise comparativa do indicador (arv/km), é imprescindível observar o tamanho amostral em cada trabalho. Pelegrim et al. (2012) observaram 106,6 árvores por quilômetro de calçada no bairro Chapadão do Sul (MS) e Malavasi et al. (1995) 114 arv/km em um bairro central de Itaguaí (RJ). Outros autores encontram resultados inferiores a 50 arv/km, todavia utilizaram um universo amostral maior do que um bairro (MILANO, 1988; RACHID; COUTO, 1999; MELLO et al., 2006; PAIVA et al., 2010; ALVAREZ; GALLO, 2012). Esse indicador espacialmente explícito, portanto, deve ser analisado e compreendido observando essas considerações e em futuros trabalhos orientar critérios previamente estabelecidos tendo em vista obter um indicador padrão (ou um conjunto) de arborização urbana.

O segundo elemento é o método empregado para quantificar os indivíduos arbóreos. Embora diversos estudos tenham demonstrado que tanto os métodos de amostragem simples como os estratificados ou por conglomerados são importantes ferramentas para a coleta de informações (com vantagens relativas do método por estratos em relação ao simples - ver MICHI; COUTO, 1996; COUTINHO; LIMA, 1997; RACHID; COUTO, 1999; MENEGHETTI, 2003; ALVAREZ et al., 2005).

A definição da unidade amostral (quarteirão ou grades regulares) pode ser um fator importante na definição da estratégia amostral antes de ir à campo coletar informações, uma vez que pode influenciar no valor do indicador, custos e tempo de campanhas de campo e precisão dos trabalhos. Diversos estudos têm demonstrado a importância e implicações do tamanho e da forma de unidades amostrais em estudos de arborização urbana (MILANO, 1988; MILANO; SOARES, 1990; MILANO et al., 1992; SILVA et al., 2005; SILVA et al., 2007; ALVAREZ et al., 2005; LIMA NETO; BIONDI, 2012). Na presente análise, foram considerados os resultados de Milano (1984; 1988), que utilizou grades regulares de diferentes tamanhos nas cidades do Paraná - Curitiba e Maringá, mas estudos mais detalhados podem contribuir para um melhor delineamento amostral que permita realizar, com efeito, as comparações.

O terceiro elemento se refere ao aspecto qualitativo. Tendo em vista a melhoria efetiva do microclima, poluição do ar, sombreamento e a melhoria da distribuição de árvores e rede elétrica e outros equipamentos urbanos (CAVALHEIRO; DEL PICCHIA, 1992; ANDRADE, 2002; MENEGHETTI, 2003; VELASCO et al., 2006; CAMARA; CINTRA, 2007; 
AGUIRRE JUNIOR; LIMA, 2007; ESCOBEDO; CHACALO, 2008), é cada vez mais urgente planejar o plantio dos tipos de espécies ou indivíduos no ambiente urbano, considerando suas características (quantidade e tipos de espécies, tamanho da copa, altura e diâmetro da árvore) e cada particularidade urbanística (recuos, calçamentos, rede elétrica), geográfica, e climática da região ou cidade.

A existência de grande variedade de espécies não é garantia de melhoria ambiental. Em Águas de São Pedro (SP), município com 133 árvores/km (SILVA FILHO; BORTOLETO, 2005) - o maior valor do indicador (arv/km) na presente análise -, apesar de apresentar maior riqueza ou variedade maior em relação a outras cidades, mostra que há uma tendência de plantio de espécies arbustivas no município que é preocupante e não garante uma diversidade ideal (BORTOLETO et al., 2007; BORTOLETO; SILVA FILHO, 2008) na proporção adequada de espécies para a arborização urbana (varia entre 10-15\% do total de espécies - GREY; DENEKE, 1978; MILANO; DALCIN, 2000; MENEGHETTI, 2003). Além disso, uma grande quantidade (ou alta densidade) de árvores nas calçadas não caracteriza uma arborização urbana bem planejada, sendo necessário considerar fatores como a dimensão e a qualidade da copa da árvore (PAIVA; GONÇALVES, 2002; ALMEIDA; RONDON NETO, 2010b).

Um quarto elemento se refere à característica dos bairros distribuídos nas cidades, que varia em função de padrões construtivos, do tipo de uso urbano (residencial, comercial e/ou industrial). $\mathrm{Na}$ presente análise pôde-se observar tanto em intervalos $>$ de 100 árvores $/ \mathrm{km}$ quanto em intervalos < de 40 árvores $/ \mathrm{km}$ a heterogeneidade dos tipos de bairros e padrões de construção, com influência nos valores de árvores por quilômetro em função do lugar. Rocha et al. (2004) concluem que o bairro Rancho Novo (residencial), em Nova Iguaçu-RJ, possui melhor qualidade de sua arborização em relação ao Centro (com característica comercial). Situações semelhantes foram encontradas em Piracicaba-SP (MICHI; COUTO, 1996), Campos do Jordão-SP (ANDRADE, 2002), Campos de Goytacazes-RJ (PEDLOWSKI et al., 2002), Taubaté-SP (MINHOTO et al., 2009), Jataí-GO (BARROS et al., 2010), Campinas-SP (ALVAREZ; GALLO, 2012).

$\mathrm{Na}$ verdade, sempre que possível, é necessário analisar caso a caso. Diversos trabalhos têm realizado as análises observando critérios de manutenção de árvores em vias públicas, diversidade de espécies em inventários, composição e estrutura das árvores (MILANO, 1984, 1988; ANDRADE, 2002; MENEGHETTI, 2003; PAIVA, 2009; PAIVA et al., 2010; PELEGRIM et al., 2012; ALVAREZ, 2004; ROCHA et al., 2004), aspectos fundamentais no escopo de um plano de arborização. 
Além disso, o processo de ocupação das cidades há décadas tem ocorrido sem se planejar adequadamente os espaços verdes em áreas edificadas, salvo exceções de cidades planejadas em conjunto com a arborização urbana e espaços verdes ou que têm buscado desenvolver seus planos diretores de arborização urbana - PDAUs (MILANO 1984; MILANO, 1988; BIONDI; ALTHAUS, 2005; SANCHES et al., 2008; BOBROWSKI et al., 2012). Se por um lado há uma estrutura ou modo de se pensar e planejar os espaços públicos e construídos que poucas vezes têm considerado uma articulação entre os instrumentos de planejamento, por outro, tem aumentado os estudos e técnicas de arborização, sobretudo com o auxílio de SIGs, análises espaciais e técnicas de sensoriamento remoto (SANTIN, 1999; NOWAK; O'CONNOR, 2001; ALVAREZ, 2004; ALVAREZ et al., 2010; CONWAY; URBANI, 2007; VELASCO et al., 2007; BARBIN et al., 2008; VIEIRA; BIONDI, 2008; LIMA; VIEIRA, 2009; LIMA NETO; BIONDI, 2012; LIMA NETO et al., 2012; ALVAREZ; GALLO, 2012). Essas técnicas têm sido aperfeiçoadas e cada vez mais utilizadas em trabalhos de arborização urbana, mostrando um potencial a ser explorado e que pode auxiliar na articulação e integração de planos urbanísticos (planos diretores e afins) com planos de arborização urbana.

Esses resultados mostram o potencial da aplicação de métodos de amostragem para estudos de arborização urbana. Também indicam que 0 uso de técnicas de geoprocessamento e sensoriamento remoto (imagens de média e alta resolução, fotografias aéreas), bem como organização de um banco de dados relacional e análises espaciais em um SIG são fundamentais para um pré e pós planejamento da arborização (SILVA FILHO, 2002; SILVA FILHO et al., 2002; SILVA et al., 2007; LIMA NETO; BIONDI, 2012)

\section{CONCLUSÕES}

O indicador árvores por quilômetro de calçada baseado na aplicação da amostragem simples nas duas cidades do oeste paulista forneceu elementos quantitativos para contribuir ao planejamento da arborização urbana nas cidades analisadas. Esses resultados incorporados em um SIG e analisados espacialmente com outros estudos podem trazer um importante instrumento para o planejamento regional das cidades brasileiras, respeitando a heterogeneidade de cada caso. Finalmente, o uso de técnicas de geoprocessamento e sensoriamento remoto (imagens de média e alta resolução, fotografias aéreas), bem como organização de um banco de dados relacional e análises espaciais em um SIG são fundamentais para um pré e pós planejamento da arborização. 


\section{AGRADECIMENTOS}

Ao apoio dos convênios entre as prefeituras de Martinópolis-SP e Pirapozinho-SP e FCT-UNESP (01350/2004; 02150/2006). Ao José R. F. Castilho, Márcio R. Pontes e Denis G. Amorim, fundamentais na realização dos diagnósticos de planos diretores no âmbito dos convênios e revisão do texto. Ao Mário H. Tarumotopela orientação estatística.

\section{REFERÊNCIAS}

AGUIRRE JUNIOR., J. H.; LIMA, A.M.L.P. Uso de árvores e arbustos em cidades brasileiras. RevistaSBAU, v.2, n.4, p. 50-66, 2007.

ALMEIDA, D.N.; RONDON NETO, R.M. Análise da arborização urbana de três cidades da região norte do Estado de Mato Grosso. Acta Amazonica, v.40, p.647-656, 2010a.

ALMEIDA, D.N.; RONDON NETO, R.M. Análise da arborização urbana de duas cidades da região norte do estado de Mato Grosso. Árvore, v.34, p.899-906, 2010b.

ALVAREZ, I.A. Qualidade do espaço verde urbano: uma proposta de índice de avaliação. Tese (doutorado em Agronomia/Fitotecnia), ESALQ/USP, Piracicaba, 2004,187p.

ALVAREZ, I.A.; VELASCO, G.D.N.; BARBIN, H.S.; LIMA, A.M.L.P.; COUTO, H.T.Z. Comparison of two sampling methods for estimating urban trees density. Journal of Arboriculture, v. 31, n.5, p. 209-214, 2005.

ALVAREZ, I.A.; SILVA FILHO, D.F.; COUTO, H.T.Z.; POLIZEL, J.L. Comparação entre videografia e fotografia aérea para diagnóstico da vegetação em ambiente urbano de Piracicaba, SP. Árvore, v. 34, p.377-384, 2010.

ALVAREZ, I.A.; GALLO, B.C. Quantificação da arborização urbana viária de Campinas, SP. Campinas: Embrapa Monitoramento por Satélite. Comunicado Técnico, n.30, , p.1-8, 2012.

ANDRADE, T.O. Inventário e análise da arborização viária da Estância Turística de Campos de Jordão, SP. Dissertação (Mestrado) - ESALQ/USP, Piracicaba-SP, 2002, $112 p$.

BARBIN, H.S.; VELASCO, G.N.; ALVAREZ, I.A.; LIMA, A.M.L.P. Estudo dos espaços livres do bairro Santa Cecília, em Piracicaba, SP. Revista SBAU, v.3, p.17-35, 2008. 
BARROS, E.F.S.; GUILHERME, F.A.G.; CARVALHO, R.S. Arborização urbana em quadras de diferentes padrões construtivos na cidade de Jataí. Árvore, v. 34, p.287-295, 2010.

BEATLEY, T.; MANNING, K.The Ecology of Place.Planning for Environment, Economy and Community.Island Press, Washington, DC, 1997.

BIONDI, D.; ALTHAUS, M. Árvores de rua de Curitiba: cultivo e manejo. Curitiba: FUPEF, 2005. $182 \mathrm{p}$.

BOBROWSKI, R.; BIONDI, D.; FIGUEIREDO FILHO, A. Dinâmica da distribuição diamétrica na arborização de ruas da cidade de Curitiba, Paraná, Brasil. ScientiaForestalis (IPEF), v.40, p.167-178, 2012.

BOLUND, P.; HUNHAMMAR, S. Ecosystem services in urban areas. Ecological Economics, n.29, p. 293-301, 1999.

BORTOLETO, S.; SILVA FILHO, D.F.; SOUZA, V.C.; FERREIRA, M.A.P.; POLLIZEL, J.L.; RIBEIRO, R.C.S. Composição e distribuição da arborização viária da Estância de Águas de São Pedro-SP. Revista SBAU, v.2, p. 32-46, 2007.

BORTOLETO, S.; SILVA FILHO, D.F. Situação da arborização viária da Estância de Águas de São Pedro - SP. Agronegócio e Meio Ambiente, v. 1, p. 391-403, 2008.

CAMARA, R.; CINTRA, D.P. Arborização viária e conflitos com equipamentos urbanos no bairro da Taquara, RJ. Floresta e Ambiente, v. 14, p. 25-33, 2007.

CAMPO GRANDE - Prefeitura Municipal de Campo Grande-MS. Plano Diretor de Arborização Urbana (PDAU). SEMADUR: Campo Grande-MS, 2010. 145 p.

CAVALHEIRO, F.; DEL PICCHIA, P. Áreas verdes: conceitos, objetivos e diretrizes para o planejamento. In: $1^{\circ}$ Congresso Brasileiro Sobre Arborização Urbana e $4^{\circ}$ Encontro Nacional Sobre Arborização Urbana. Anais... Vitória-ES,v. 1, p. 29-38, 1992.

COCHRAN, W.G. Samplingtechniques. $3^{\text {ed }}$. New York: John Wiley, 1977. 428p.

CONWAY, T.M.; URBANI, L. Variations in municipal urban forestry policies: A case study of Toronto, Canada. UrbanForestry\&UrbanGreening, v.6, p.181-192, 2007.

COSTA, L.A.C.; HIGUCHI, N. Arborização de ruas de Manaus: avaliação qualitativa e quantitativa. Árvore, v.23, n.2, p.223-232, 1999.

COUTINHO, C.L.; LIMA, J.P.C. Métodos de amostragem para avaliação de arborização viária. Floresta e Ambiente, v. 4, p.110-116, 1997. 
DOMBROW, J.; RODRIQUEZ, M.; SIRMANS, C.F.The market value of mature trees in single family housing markets.Appraisal Journal, n.68, p.39-43, , 2000.

ESCOBEDO, F.; CHACALO, A. Estimación preliminar de ladescontaminación atmosférica por elarbolado urbano de laciudad de México. Interciencia, v.33, n.1, 2008.

GRAZIANO, T. T. Viveiros Municipais. Departamento de Horticultura - FCAVJ - UNESP. Notas de Aula, 1994.

GREY, C.; DENEKE, F. Urban Forestry. John Wiley.Chichester, 1978, 279 p.

LAURIE, I. Nature in Cities. The Natural Environment in the Design and Development of Urban Green. John Wiley: Chichester, 1979, 428 p.

LIMA, P.C.F.; OLIVEIRA, V.R.; NASCIMENTO, C.E.S.; TORRES, S.B. Diagnóstico da arborização de ruas de Petrolina-PE. In: Encontro Nacional sobre Arborização de Ruas de Petrolina-PE. Anais... Curitiba-PR, p.41-53, 1990.

LIMA, M. O.; VIEIRA, V.C.B. Uso de Geotecnologias para Análises da Cobertura Vegetal Urbana. In: XIV SBSR. Anais... Natal-RN, p. 731-738, 2009.

LIMA NETO, E. M.; BIONDI, D. Delineamento espacial de unidades amostrais para o inventário da arborização de ruas em Curitiba, PR. Revista SBAU, v.7, p.107-118, 2012.

LIMA NETO, E. M.; BIONDI, D.; ARAKI, H.; BOBROWSKI, R. Fotografias aéreas para mensuração da área de copa das árvores de ruas de Curitiba-PR. Floresta, v. 42, p. 577588, 2012.

MALAVASI, U.C.; SOBRINHO, J.A.; GAMA, L.L.M.F.; ANDRADE, A.G.; ROCHA, A.R. A arborização urbana nacidade de Itaguaí-RJ. Floresta e Ambiente, v.2, p.74-77, 1995.

MARANHO, A.S.; PAULA, S.R.P.; LIMA, E.; PAIVA, A.V.; ALVES, A.P.; NASCIMENTO, D.O. Levantamentocensitário da arborização urbana viária de Senador Guiomard, Acre. Revista SBAU, v. 7, p. 44-56, 2012.

MASCARÓ, L. Ambiência Urbana. Sagra-Luzzatto, Porto Alegre (RS), 1996.

McPHERSON, G.E.; SIMPSON, J.R.; PEPER, P.J.; MACO, S.E.; XIAO, Q. Municipal forest benefits and costs in five US cities. Journal of Forestry, v.103, n.8, p.411-416, 2005.

MENEGHETTI, G.I.P. Estudo de dois métodos de amostragem para inventário da arborização de ruas dos bairros da orla marítima do município de Santos, SP. Dissertação (mestrado) - ESALQ/USP, 2003. 100 p. 
MELLO, A.Y.I.; TARUMOTO, M. H.; PONTES, M.R.; AMORIM, D.G. Aplicação de método de amostragem para arborização urbana. In: XVIII Congresso de Iniciação Científica. Anais...Bauru/SP, 2006.

MICHI, S.M. P.; COUTO, H.T.Z. Estudo de dois métodos de amostragem de árvores de ruanacidade de Piracicaba, SP. In: 1ํ Curso emTreinamento sobre Poda emEspécies Arbóreas Florestais e de Arborização Urbana. Anais...Piracicaba: IPEF, v.1. p.1-23, 1996.

MILANO, M.S. Avaliação e análise da arborização de ruas de Curitiba-PR. Dissertação (Mestrado) - UFPR, Curitiba-PR, 1984, 130 p.

MILANO, M. S. Avaliação Quali-Quantitativa e manejo da Arborização Urbana: exemplo de Maringá-PR. Tese de Doutorado, UFPR, Curitiba-PR, 1988, 120 p.

MILANO, M. S.; SOARES, R.V. Aplicação de técnicas de amostragem aleatória para avaliação de ruas de Maringá (PR). In: Encontro Nacional de Arborização Urbana. Anais... Curitiba-PR, p. 244-251, 1990.

MILANO, M. S.; SARNOWSKI FILHO, O.; MEZAROBAYO, J.A. Estudo comparativo de unidades amostrais utilizadas para inventário quali-quantitativo de arborização de ruas em Curitiba. . In: I Congresso Brasileiro sobre Arborização Urbana. Anais...Vitória-ES, p. 343350, 1992.

MILANO, M. S.; DALCIN, E. S. Arborização de vias públicas. Rio de Janeiro: LIGHT, 2000. 206p.

MILLER, R.W. UrbanForestry. Planning and Managing Urban Green Spaces. Prentice Hall.Englewood Cliffs.New Jersey, 1988, 404p.

MINHOTO, E.S.; MONTEIRO, E.A.; FISCH, S.T.V. Arborização Viária na Cidade de Taubaté, SP: no Centro Comercial Histórico e um Bairro Residencial Moderno. Revista SBAU, v.4, n.2, p.82-96, 2009.

NOWAK, D.J.; O'CONNOR, P.R. Syracuse urban forest master plan: guiding the city's forest resource into the 21st century. Gen. Tech.Report NE-287.U.S., 2001.50 p.

NOWAK, D.J.; DWYER, J.F. Understanding the benefits and costs of urban forest ecosystems.In: Kuser, J.E. (Ed.). UrbanandCommunityForestry in theNortheast. $2^{\text {ed. }}$, Springer, Dordrecht, p. 25-45, 2007.

PAIVA, H. N.; GONÇALVES, W. Florestas urbanas: planejamento para melhoria da qualidade de vida. Viçosa: Aprenda Fácil, 2002. 180 p. 
PAIVA, A.V. Aspectos da Arborização Urbana do Centro de Cosmópolis-SP. Revista SBAU, v.4, n.4, p.17-31, 2009.

PAIVA, A.V.; LIMA, A.B.M.; CARVALHO, A.; JUNIOR, A.M.; GOMES, A.; MELO, C.S. et al. Inventario e Diagnóstico da Arborização Urbana Viária de Rio Branco, AC. Revista SBAU, v. 5, n.1, p.144-159, 2010.

PAYNE, B.R.; STROM, S.The contribution of trees to the appraised value of unimproved residential land.Valuation, v.22, p.36-45, 1975.

PEDLOWSKI, M.A.; SILVA, V.A.C.; ADELL, J.J.C.; HEYNEN, N.C. Urban Forest and environmental in equality in Campos dos Goytacazes, Rio de Janeiro, Brazil. UrbanEcosystem, v.6, p.9-20, 2002.

PELEGRIM, E.A.L.; LIMA, A.P.L.; LIMA, S.F. Avaliação qualitativa e quantitativa da arborização no bairro Flamboyant em Chapadão do Sul, MS. Revista SBAU, v.7, p.126-142, 2012.

RABER, A.P.; REBELATO, G.S. Arborização Viária do Município de Colorado, RS-Brasil: Análise quali-quantitativa. Revista SBAU, v.5, n.1, p.183-199, 2010.

RACHID, C.; COUTO, H.T.Z. Estudo da eficiência de dois métodos de amostragem de árvores de rua na cidade de São Carlos, SP. Scientia Forestalis, v.56, p.59-68, 1999.

ROCHA, R.T.; LELES, P.S.S.; OLIVEIRA NETO, S.N. Arborização de vias púbicas em Nova Iguaçu, RJ: o caso dos bairros Rancho Novo e Centro. Árvore, v.28, p.599-607, 2004.

ROSSATTO, D.R.; TSUBOY, M.S.F.; FREI, F. Arborização Urbana na Cidade de Assis: Uma Abordagem Quantitativa. Revista SBAU, v.3, n.3, p. 1-16, 2008.

SAMPAIO, A.C.F.; DE ANGELIS, B.L.D. Inventário e Análise da Arborização de Vias Públicas de Maringá-PR. Revista SBAU, v.3, n.1, p.37-57, 2008.

SANDERS, R.A. Urban vegetation impacts on the hydrology of Dayton, Ohio. Urban Ecology, v. 9, p.376-361, 1986.

SANCHES, P.M.; COSTA, J.A.; SILVA FILHO, D.F. Análise comparativa dos Planos Diretores de Arborização Enquanto Instrumento de Planejamento e Gestão. Revista SBAU, v. 3, p.53-74, 2008.

SANTIN, D.A. Vegetação remanescente do município de Campinas (SP): mapeamento, caracterização fisionômica e florística, visando a conservação. Tese (Doutorado), IB/UNICAMP, Campinas-SP, 1999. 
SANTINI, R.C.G. Dimensões do lazer e da recreação: questões espaciais, sociais e psicológicas. São Paulo: Angelotti, 1993.

SHEETS, V.L.; MANZER, C.D. Affect, cognition, and urban vegetation: some effects of adding trees along city streets. Environment and Behavior, v.23, n.3, p.285-304, 1991.

SILVA, A.G.; GONÇALVES, W.; LEITE, H.G.; SANTOS, E. Estudo do tamanho e da forma de unidades de amostra, utilizando a amostragem casual simples para inventariar a arborização urbana viária. Natureza \& Desenvolvimento, v. 1, n. 1, p. 59-66, 2005.

SILVA, A.G.; PAIVA, H.N.; GONÇALVES, W. Avaliando a arborização urbana. Coleção Jardinagem e paisagismo, v.5. Viçosa-MG: Ed. Aprenda Fácil, 2007. 346p.

SILVA FILHO, D.F. Cadastramento informatizado, sistematização e análise da arborização das vias públicas da área urbana do município de Jaboticabal, SP. Dissertação (Mestrado) - UNESP, Jaboticabal, 2002, 81p.

SILVA FILHO, D.F.; PIZETTA, P.U.C.; ALMEIDA, J.B.S.A.; PIVETTA, K.F.L.; FERRAUDO, A.S. Banco de dados relacional para cadastro, avaliação e manejo da arborização em vias públicas. Árvore, Viçosa, v. 26, n.5, p. 629-642, 2002.

SILVA FILHO, D.F.; BORTOLETO, S. Uso de indicadores de diversidade na definição de plano de manejo da arborização viária de Águas de São Pedro-SP. Árvore, v.29, p.973-982, 2005.

SOARES, M.P. Verdes Urbanos e Rurais. Editora Cinco Continentes, Porto Alegre (RS), 1998.

ULRICH, R.S. View through a window may influence recovery from surgery. Science, v.224, p.420-421, 1984.

VELASCO, G.D.N; LIMA, A.M.L.P.; COUTO, H.T.Z. Análise Comparativa dos custos de diferentes redes de distribuição de energia elétrica no contexto da arborização urbana. Árvore, v.30, p.679-686, 2006.

VELASCO, G.D.N.; POLIZEL, J.L.; COLTRI, P.P.; LIMA, A.M.L.P.; SILVA FILHO, D.F. Aplicação do Índice de Vegetação NDVI (NormalizedDifferenceVegetation Index) em Imagens de Alta Resolução no Município de São Paulo e suas limitações. Revista SBAU, v.2, p.1-12, 2007.

VIEIRA, C.H.S.D.; BIONDI, D. Análise da dinâmica da cobertura vegetal de Curitiba, PR (de 1986 a 2004), utilizando imagens Landsat TM. Árvore, v.32, n.3, p.479 - 487, 2008. 